\title{
Shifts in Contraceptive Use in the City of Tehran, Iran: 2000-2014
}

\author{
Amir Erfani ${ }^{*}$, Javad Shojaei ${ }^{2}$
}

1. Professor, Department of Sociology \& Anthropology, Nipissing University,North Bay, Canada

2. Senior Expert (PhD.), Population Policy Studies and Monitoring Committee, Supreme Council for Cultural Revolution, Tehran, Iran

Article Info

Received: 2019/04/07;

Accepted: 2019/11/12;

Published Online: 2020/01/03

$\underline{10.30699 / \text { ajnmc. } 28.1 .56}$

Original Article

Use your device to scan and read the article online

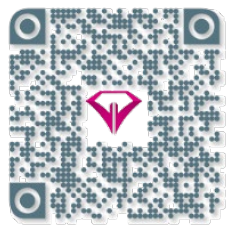

\section{ABSTRACT}

Introduction: Current data on changes in contraceptive use is required to prevent unintended pregnancies. This study aims to study trends, and patterns of contraceptive use from 2000 to 2014.

Methods: This survey research uses data from three fertility surveys conducted in 2000, 2009, and 2014 respectively among representative samples of 1396, 2,934 and 3012 married women aged 15-49 living in the capital city of Tehran. The prevalence of contraceptive methods was estimated according to women's socioeconomic and demographic characteristics.

Results: In $2014,82 \%$ of women used contraceptive methods ( $48 \%$ modern methods, and 34\% traditional methods). Withdrawal (33\%) and condoms (21\%) had the highest prevalence, compared with female sterilization (9\%), IUD (7\%) and Pills (7\%). Over 2000-2014, the use of withdrawal and condoms increased by $69 \%$ and $20 \%$, respectively. In contrast, the prevalence of female sterilization, pills, male sterilization, and IUD decreased respectively by $20 \%, 42 \%, 45 \%$ and $51 \%$ over the same period. The use of withdrawal method increased with women's age. Withdrawal and condom were used by $67 \%$ of contraceptive users, who mostly hold higher education levels, were employed, and lived in upper residential districts. In contrast, users of modern methods, namely sterilizations, IUD and pills, mostly hold lower levels of education and income, were unemployed, lived in the lower residential districts, and had two or more children.

Conclusion: The increasing prevalence of withdrawal and condom signifies a need for provision of effective family planning education and counseling to prevent unintended pregnancy.

Keywords: Contraception; Family planning; Reproductive health

\section{How to Cite This Article:}

Erfani A, Shojaei J. Shifts in Contraceptive Use in the City of Tehran, Iran: 2000-2014. Avicenna J Nurs Midwifery care. 2020; 28 (1):56-66 
تحولات استفاده از روشهاى ييشگيرى از باردارى در سالهاى

امير عرفانى ا";، جواد شجاعى

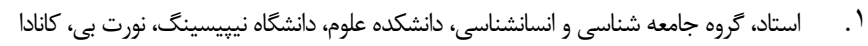

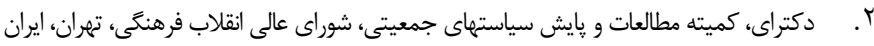

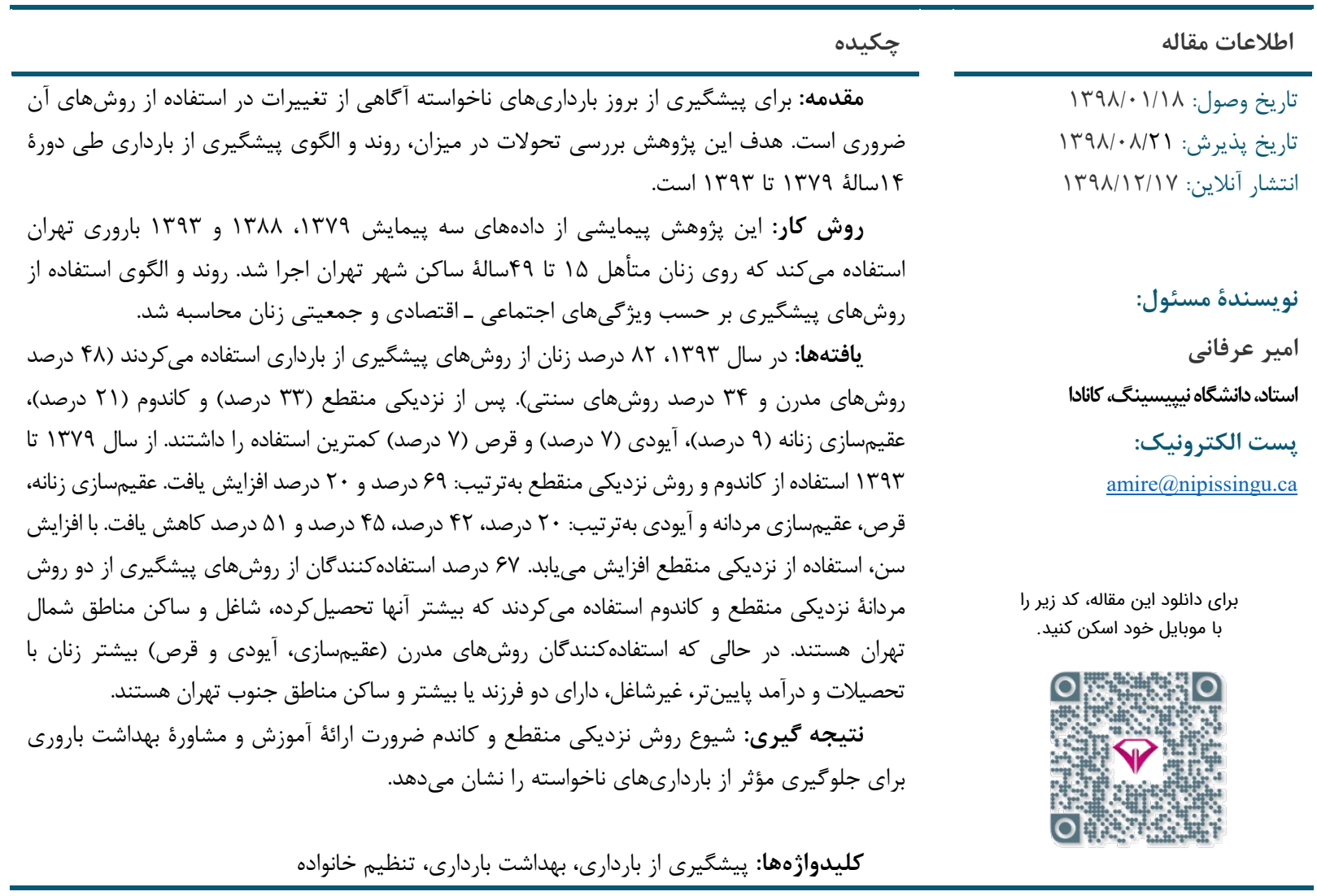

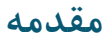

با وجود اين در دو دهأ اخير به واسطؤ تداوم ميزان ميزان

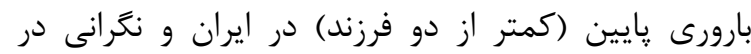

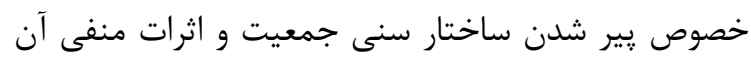

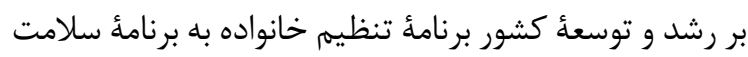

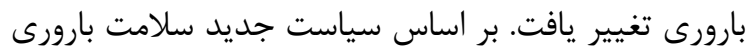

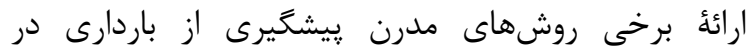
واحدهاى بهداشتى ـ درمانى دولتى كشور محدود (مانند بردي عقيمسازى زنانه و آيودى) يا ممنوع (مانند عقيمسازى دئى داني

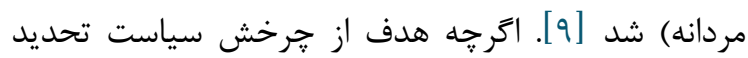
مواليد به سياست تشويق مواليد ارتقاى ميزان مواليد اعلام

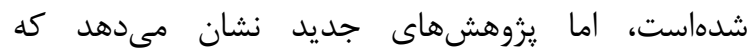

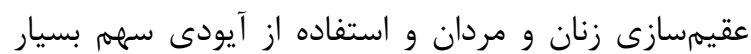

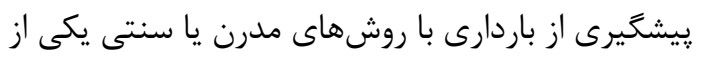

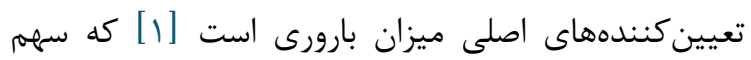

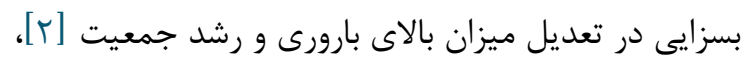
بهبود سلامت كودى و مادر، با فاصله كذارى مناسب باري بين

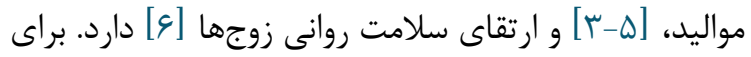

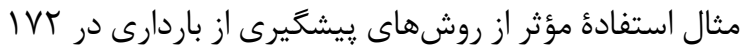

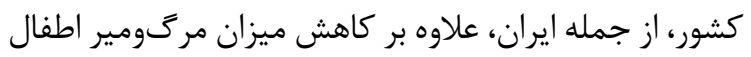

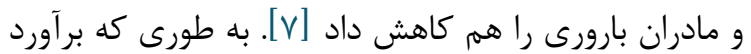

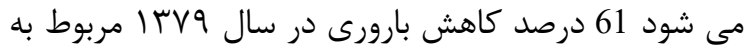

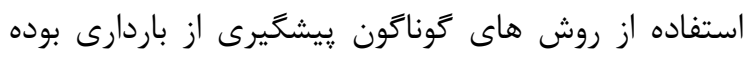

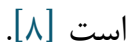


شد. با استفاده از روش نمونهَيرى احتمالى دو مرحلهاى

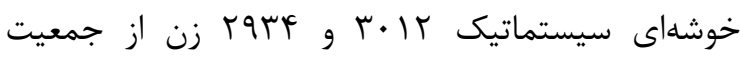

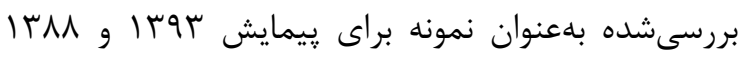

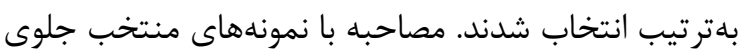

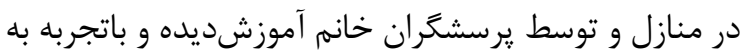
صورت جهرهبهجهره انجام شد. جزييات روش نمونه

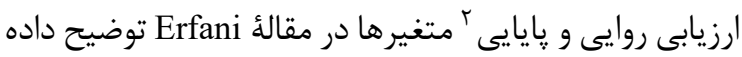

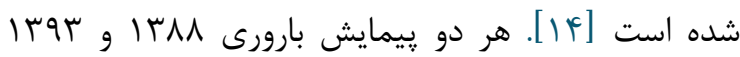

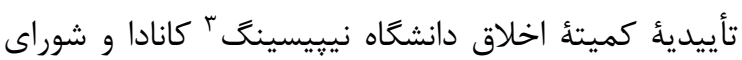

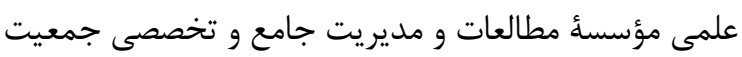
كشور در ايران را دريافت كردهاند. ي يمايش اما براى اين يزوهش فقط از دادههاى مربوط به شهر تهران

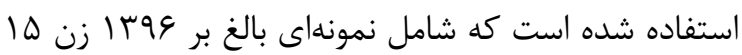

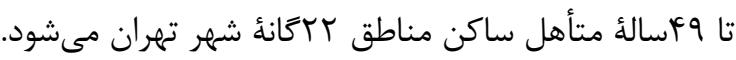

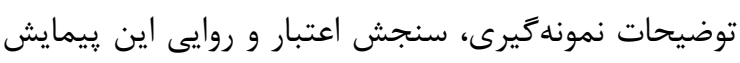

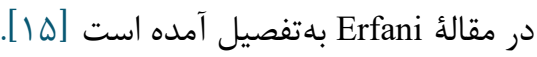

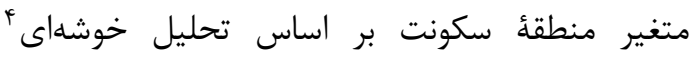

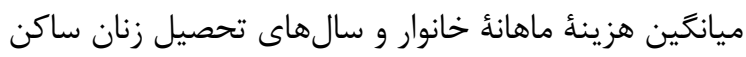

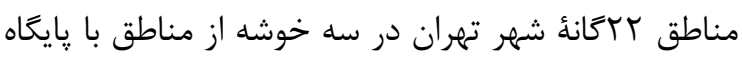

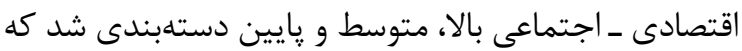
در اين مقاله استفاده مىشود.

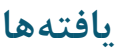

\section{ويثزى هاى نمونه}

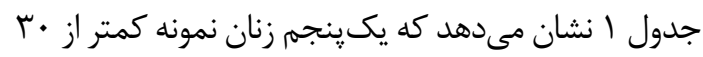
سال دارند و بيش از دوسوم آنها يُ إن از 9 اسالكَى ازدواج نداج كردهاند. بيش از 91 درصد افراد نمونه باسوادند. به طورى كه آنه

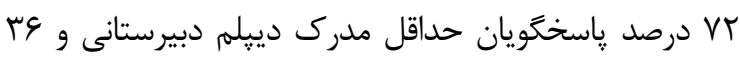

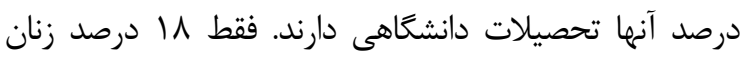
متأهل در سن بارورى و ساكن شهر تهران در زمان ماند مصاحبه

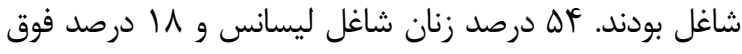

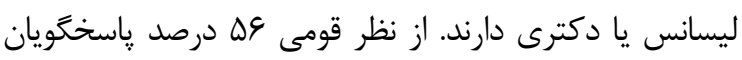

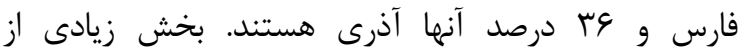
ياسخكويان در مناطق مركزى و جنوبى شهر تهران زندگى

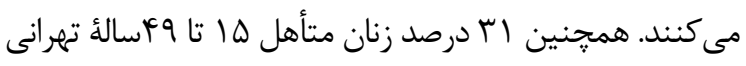

\footnotetext{
${ }^{3}$ Nipissing

${ }^{4}$ Cluster analysis

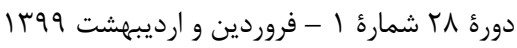

ناجيزى (V درصد) در كاهش بارورى داشته است [. []. از اين رو اجراى موفق هر كونه سياست جمعيتى تشويق مواليد

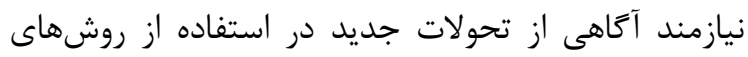
ي بيشَيرى از مواليد است.

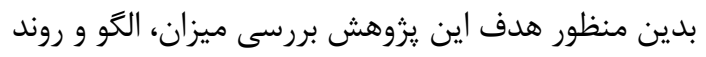

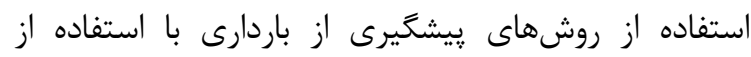

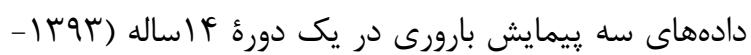
أن IFV9

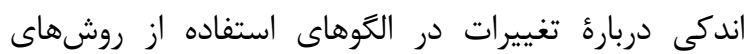

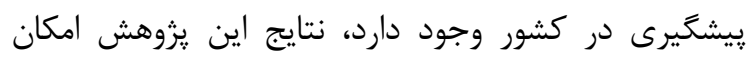

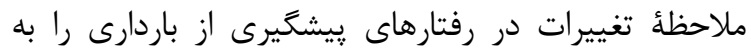

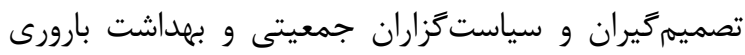

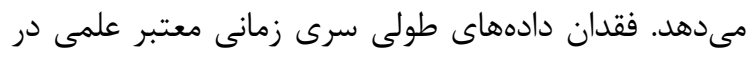

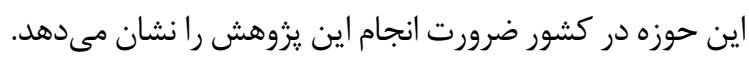

\section{روش بررسى}

اين يزوهش عمدتا از دادهاى ييمايش بهوبا بارورى

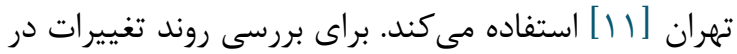

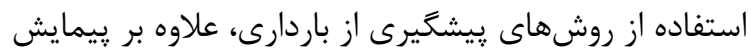

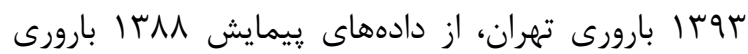

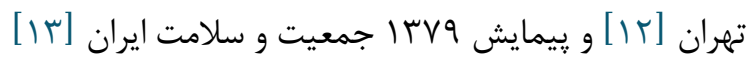
استفاده شده است.

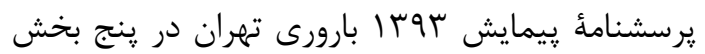

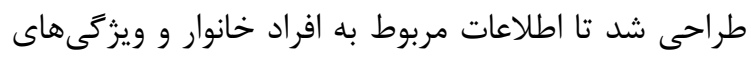

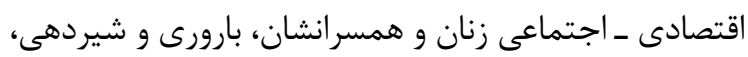

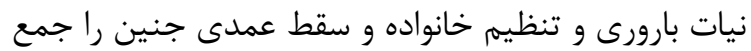

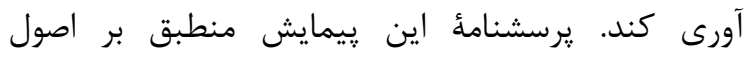

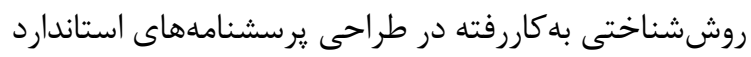
ييمايشهاى معروف جمعيت و بهداشت' است. همجنين برخى يرسشهاى ييمايش ITV9 جمعيت و بهداشت ايران

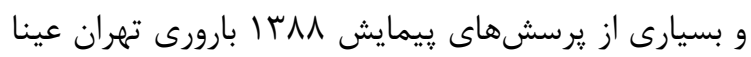

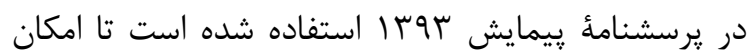
مقايسٔ روند تغييرات شاخصهاى جمعيتى و بهداشتى، از بران جمله بيشخيرى از باردارى، فراهم شود.

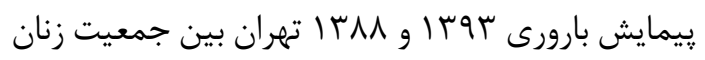

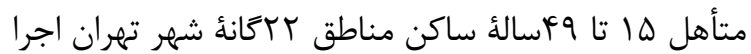

${ }^{1}$ Demographic and Health Surveys (DHS)

${ }^{2}$ Validity and reliability 
همين دوره ميانگين سن ازدواج زنان در تهران ه/ ال سال افزايش كان يافته است. در حالى كه در اين دوره كمتر از يك سال (A/.

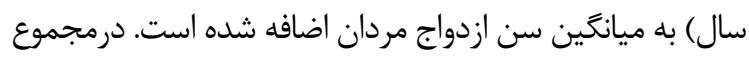
سطح تحصيلات زنان متأهل و شوهرانشان در اين دوره ينجساله يك سال افزايش يافته است.
مهاجر هستند. به طورى كه r T درصد آنها از شهرها و 9 درصد از روستاها به تهران مهاجرت كردهاند.

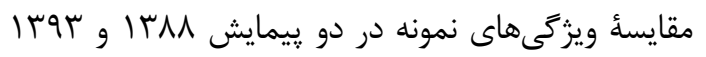

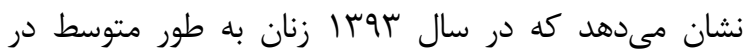

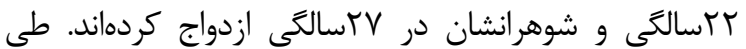

جدول ا. توزيع فراوانى و درصد زنان متأهل ها تا q\&اساله بر حسب ويثَى هاى زمينهاى سال بqسا در شهر تهران

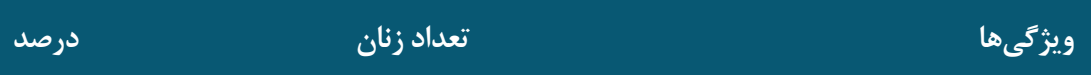

\begin{tabular}{|c|c|c|}
\hline$\cdot / V$ & Tr & $19-10$ \\
\hline$\Delta / 1$ & $\mid Q F$ & $r F-r$. \\
\hline $\mid \Delta / 1$ & FDS & $r q-r \Delta$ \\
\hline$r \Delta / r$ & $V \Delta q$ & $r \mu_{-} r$. \\
\hline $19 / 9$ & $4 .$. & ט \\
\hline$I V / V$ & DrT & $p q-F$. \\
\hline $19 / \pi$ & $4 \wedge 9$ & $r q-i \Delta$ \\
\hline
\end{tabular}

$9 / 9$

G/V r.r

$1 \cdot 10$

rV/T IITT

V/S Trg

rT/9 919

$\Delta / 1$

$\Lambda T / 1$

IV/9 $\Delta r \Lambda$

$1 \cdot / 4 \quad$ mIF

TI/F GFT

FT/T ITVD

$r \Delta / 9$

$\Delta \mathrm{Q} / \mathrm{A}$

$r \cdot / r$

G/V

$r / Q$

$\Gamma / \Lambda$

$\Delta F / V$

$r \Psi /$

$I T / K$

$r \mu / r$
91 .

$r \cdot r$

$1 \cdot 0$

111

IGYA

११५

rVI

$v \cdot 1$

\section{سطح تحصيلات (سالهاى تحصيل)}

ابتدايى و كمتر (ه-•)

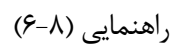

دبيرستان (II- (1) (1) (1) (1)

دييلم (IT)

كاردانى (f)

كارشناسى (19-هانى (1)

كارشناسى ارشد و بالاتر (V)

وضعيت اشتغال

غيرشاغل

شاغل

سن ازدواج

19 سال و كمتر

$1 V-19$

t. - th

فاسل و بيشتر

قوميت

فارس - توميت

ترك

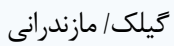

لر

كرد/ ساير

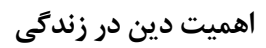

خيلى مهرم است

تاحدى مهم است

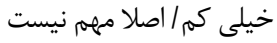

جارك هزينه خانوار (•... تومان)

كمتر از ... 


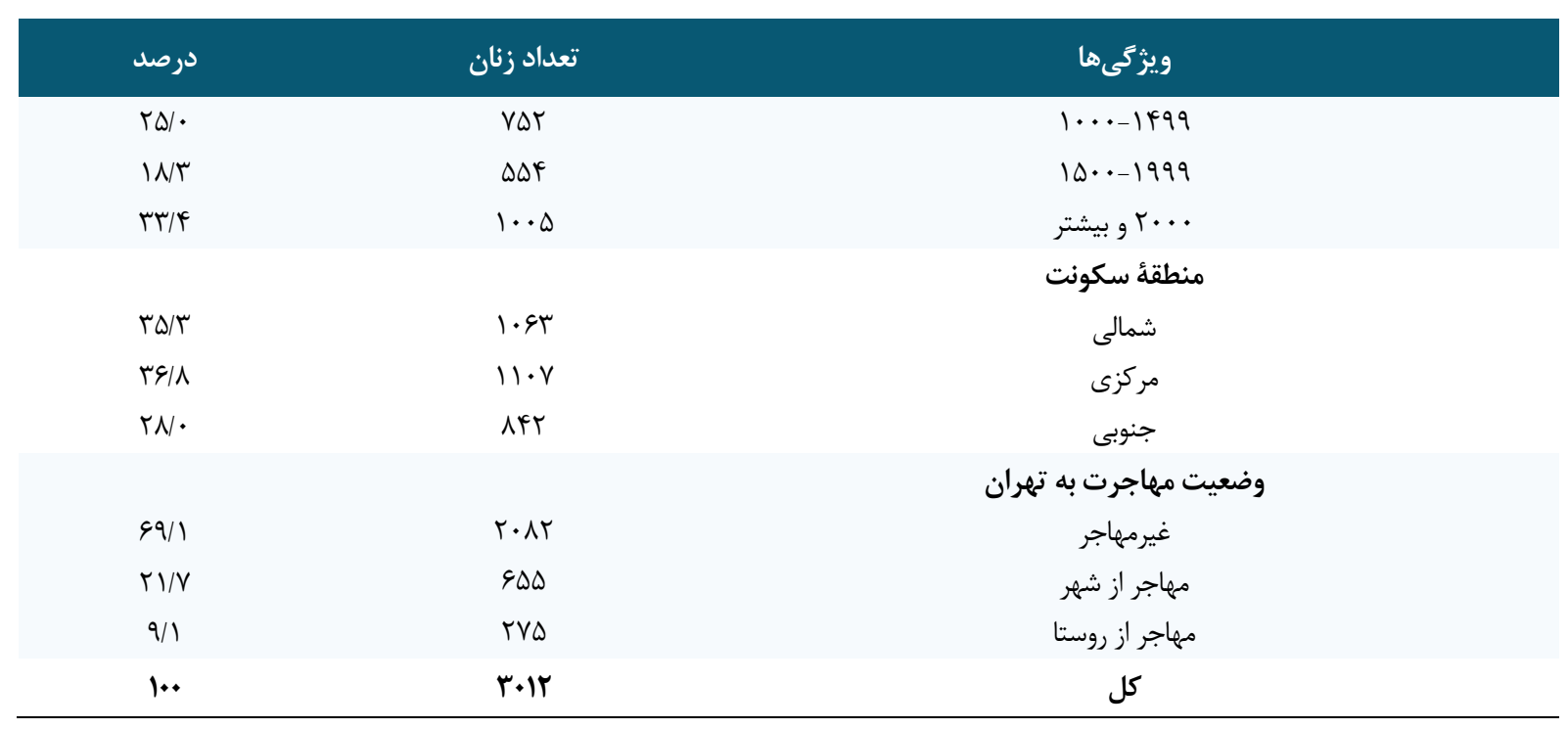

مدرن و M M درصد از روشهاى سنتى استفاده مىكنند.

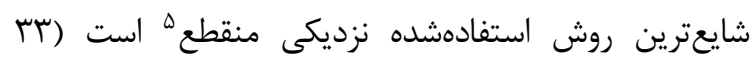
درصد). بين روشهاى مدرن كاندوم (الr درصد)، عقيمسازى روسئ زنان (9 درصد)، آيودى (V درصد) و قرص (V د درصد) بلهترتيب بيشترين ميزان استفاده را دارند. (اندان
ميزان و الكوى استفاده از روشهاى ييشگيرى جدول r توزيع درصدى زنان متأهل را بر حسب استفاده از

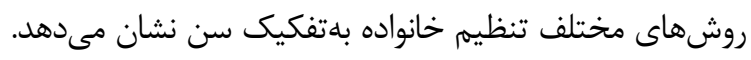

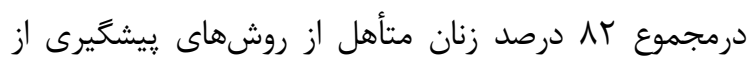

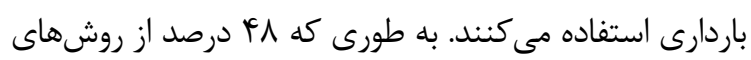

جدول r. توزيع درصد زنان متأهل ها تا qFاسله بر حسب روش ييشگيرى استفادهده در زمان مصاحبه به تفكيك سن در سال سوسا در تهران

\begin{tabular}{|c|c|c|c|c|c|c|c|c|c|c|c|c|c|c|c|}
\hline \multicolumn{2}{|c|}{ كل } & \multicolumn{2}{|c|}{ استفاده نمىكند } & \multicolumn{2}{|c|}{ روش سنتى } & \multirow{2}{*}{ روشتى رهاى } & \multicolumn{6}{|c|}{ روشهاى ملرن } & \multirow{2}{*}{ مدرن } & \multirow{2}{*}{ روشمها } & \multirow{2}{*}{ سن } \\
\hline تعداد & درصد & روشيج & لبت & مطورئ & تزقطيكى & & كلدوم & قرص & تزريق" & آيودى & ع مقيهياز & زع زنيها & & & \\
\hline rr & $1 .$. & {$[\mathrm{TJ} \mathrm{V}$} & $\mid r / 9$ & $\%$ & rVIT & TYIY & $Y V Y F$ & $\mid r / 8$ & $*$ & $F / \Delta$ & $* \%$ & $\%$ & $F \Delta \Delta$ & $\mathrm{Vr} / \mathrm{V}$ & $10-19$ \\
\hline lof & $1 .$. & $\mid r / F$ & $1 F / 9$ &.$/ 9$ & $r / / r$ & $\mathrm{r} / \mathrm{A}$ & $r \cdot / r$ & $11 / V$ & $1 / 9$ & $V_{11}$ & 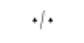 & $\%$ & $F \cdot / 9$ & $V Y / V$ & $Y \cdot-Y F$ \\
\hline$F \Delta S$ & $1 .$. & 1.11 & $11 /$. & $\cdot / f$ & $\mathrm{rr} \cdot$ & $\pi / F$ & TNY & 1.11 & $\cdot / F$ & VIr & $\cdot / f$ & $\cdot / r$ & $\varphi 9 / \Delta$ & VNG & $r Q-Y q$ \\
\hline$V \Delta 9$ & $1 .$. & $q / F$ & $V / r$ & $\cdot / \Delta$ & $r r / V$ & $r \pi / r$ & $r \cdot / \Lambda$ & GIY & $1 / 1$ & $V / \Delta$ & $1 / F$ & ५). & $\Delta \cdot 11$ & $\Delta r / r$ & $r+-r q$ \\
\hline s.. & $1 .$. & 4.1. & $r / \Lambda$ & $\cdot / r$ & $r \Delta \Lambda$ & $|r q|$. & 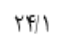 & vio & $1 / 1$ & 1.1 & Mr & $N r$ & $\Delta F / Y$ & $9 . / r$ & YQL-YQ \\
\hline$\Delta r r$ & $1 .$. & $11 / r$ & $\cdot / F$ & $\%$ & $r q / r$ & $r q / r$ & $1 . / 4$ & $\Delta / \Lambda$ & $\cdot / \Lambda$ & $N^{9}$ & $9 / 1$ & $19 / 9$ & $F q / 1$ & $M N Y$ & $F+-F F$ \\
\hline$F / 9$ & $1 .$. & Tr/Y & $\cdot / r$ & $\cdot / r$ & rVIr & $r V / F$ & $F / V$ & $r / r$ & $\cdot / r$ & $r / 1$ & $V / \Lambda$ & $r r / l$ & $f \cdot / 1$ & $s V_{i \Delta}$ & $F Q-F q$ \\
\hline$r .1 r$ & $1 .$. & $1 \pi / 1$ & $\Delta / r$ & & $r r / F$ & $\mathrm{rr} / \mathrm{V}$ & $r .19$ & $g / V$ & $\cdot / A$ & $V / F$ & $r / \Delta$ & १/. & $P N \mid$. & $\mathrm{Al} / \mathrm{V}$ & كل \\
\hline
\end{tabular}

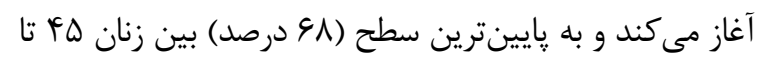

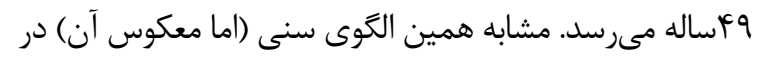

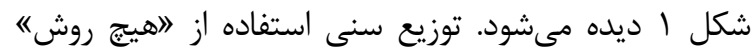

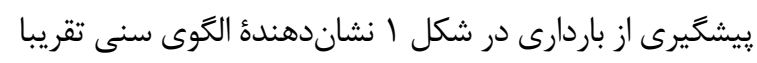

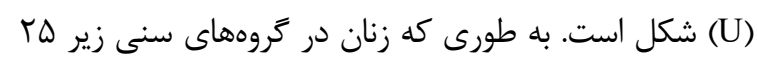

جدول r نشان مىدهد كه استفاده از همة روشهاى ييشخيرى بر حسب سن تقريبا يك الخوى U معكوس دارد. به

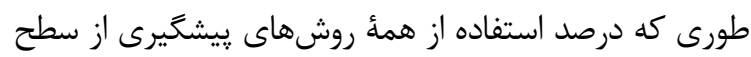

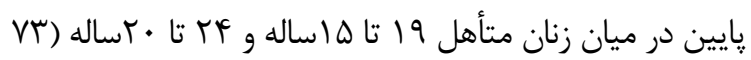

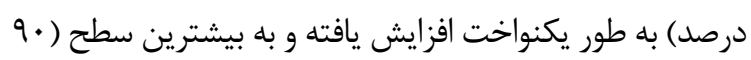

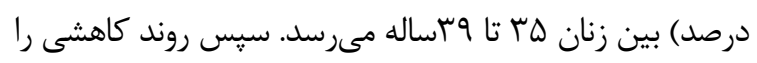

\footnotetext{
${ }^{5}$ Coitus interruptus 
استفاده از آيودى در زنان ها تا كأساله مشاهده شدهاست.

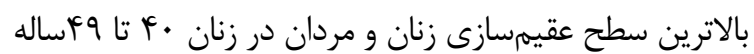

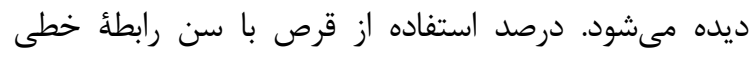

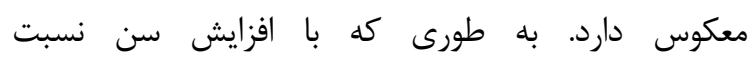

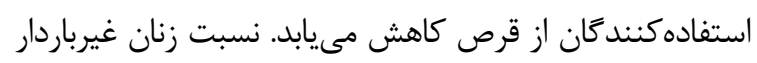

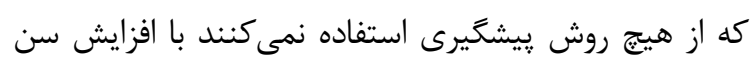

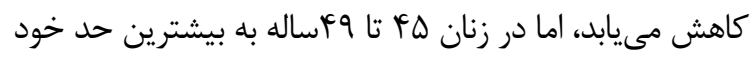

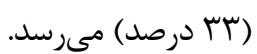

سال و بالاى FF سال بيشترين درصد استفاده نكردن از هركونه روش ييشگيرى از باردارى را نشان مى دهد.

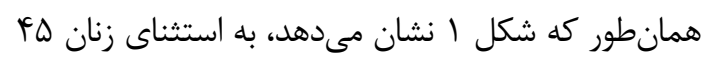

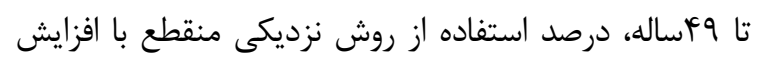
سن زنان به طور خطى افزايش مى يابد. به طورى كه استفاده از

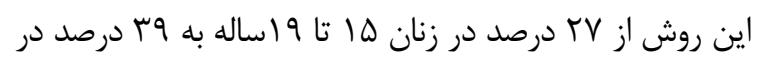

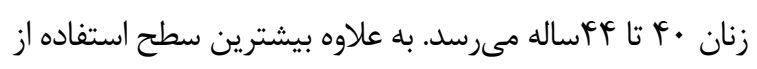

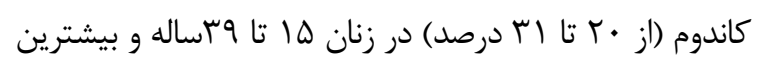

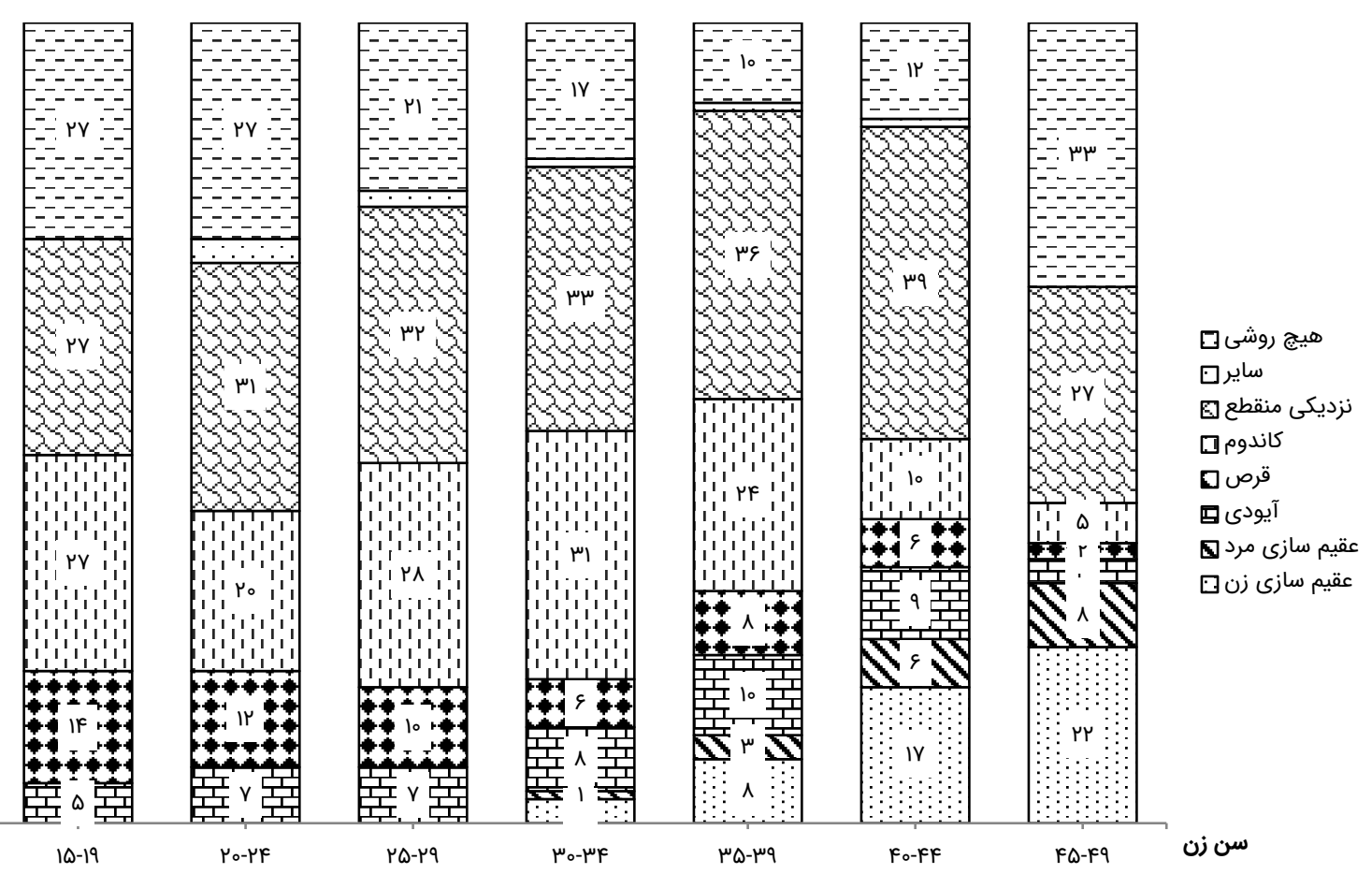

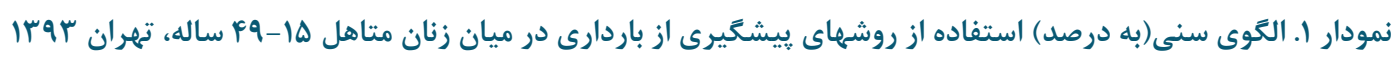

مىيابد. زنان شاغل (NF درصد) اندكى بيشتر از زنان غيرشاغل

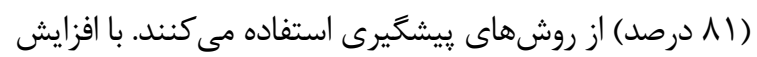

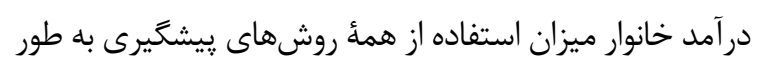

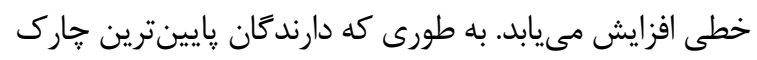

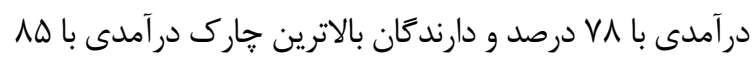

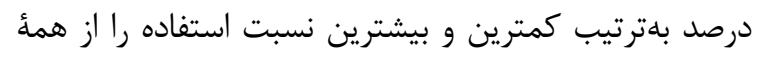

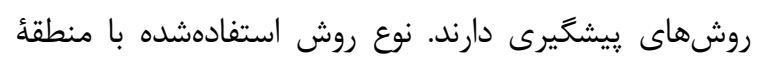

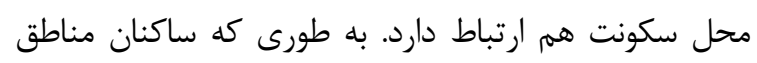

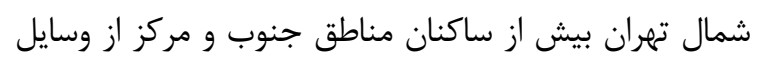
ييشخيرى استفاده مى كنند.
جدول r نشان مىدهد كه استفاده از روشهاى ييشخيرى بر حسب تعداد فرزندان زنده، سطح تحصيل، وضعيت اشتغال، در آمد، منطقة سكونت و قوميت متفاوت است. سطح استفاده از

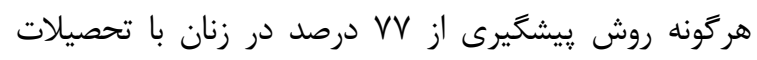

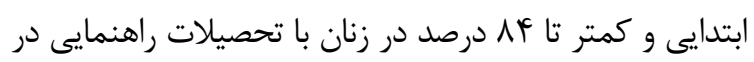

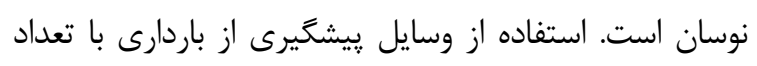

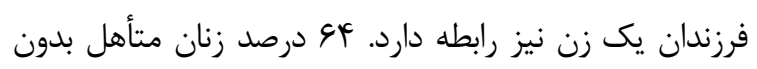
فرزند روشهاى تنظيه خانواده را براى به تعويق انداختن نئن

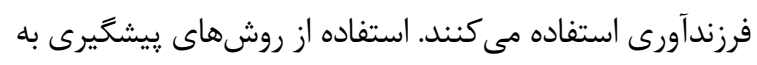

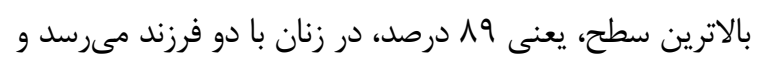

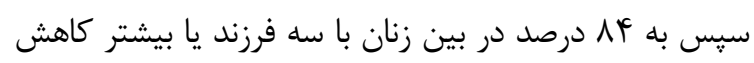


غيرمهاجران (هـ درصد) بيشترين سطح استفاده از روش نزديكى

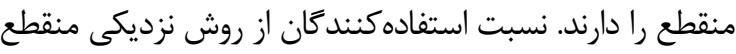
با افزايش تحصيلات و درآمد به طور خطى افزايش مى ايليد.

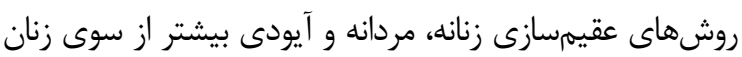
با تحصيلات و درآمد بايينتر، غيرشاغل، ساكن مناطق جنوب آنائه

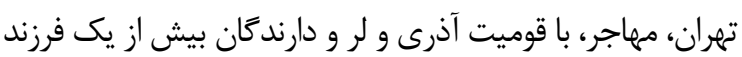

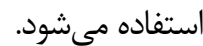

ساير نتايج بر حسب نوع روش در جدول ب نشان مىدهد كه

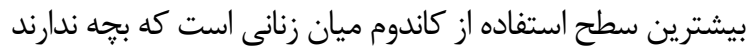

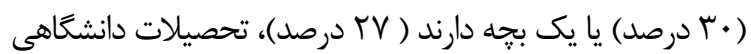

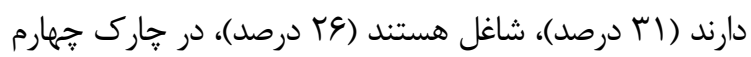

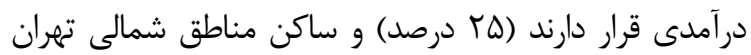

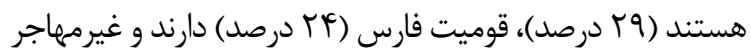

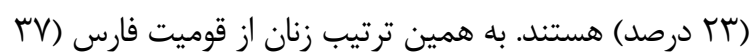
درصد) و كيلك و مازندرانى (F د درصد)، شاغلان (أF درصد)،

جدول r. توزيع درصد زنان متأهل ها تا وFاساله بر حسب روش بِيشخيرى استفادهشه در زمان مصاحبه بهتفكيك ويزگى هاى فردى سال سوبا در شهر تهران

\begin{tabular}{|c|c|c|c|c|c|c|c|c|c|c|c|c|c|c|c|}
\hline \multicolumn{2}{|c|}{ كل } & \multicolumn{2}{|c|}{ استفاده نمىكثد } & \multicolumn{2}{|c|}{ روش ستى } & \multirow[b]{2}{*}{ روشتى ستى } & \multicolumn{6}{|c|}{ روشهماى مدرن } & \multirow[b]{2}{*}{ مدوشنهاى } & \multirow[b]{2}{*}{ روشمأ } & \multirow[b]{2}{*}{ 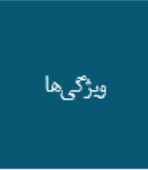 } \\
\hline تعلاد & درصد & روش هيج & باردار & 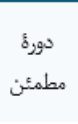 & مثزديكى & & كائدوم & قرص & تريق" & آيودى & مقرى عقيم & سازى & & & \\
\hline & & & & & & & & & & & & & & & تعداد فرزندان \\
\hline fqf & $1 \ldots$ & $r \cdot / r$ & 191 & $\cdot \pi$ & $r f / V$ & $r+q$ & rq/1 & $V / \Delta$ &.$/ 9$ & $\cdot / f$ & $\cdot / f$ & $\cdot \cdot$ & TNV & 949 & هيج \\
\hline $1 \ldots$ & $1 \cdots$ & $11 / f$ & 9) & $\cdot / r$ & TNV & rNq & rVIT & Vil & $\cdot / \Lambda$ & $9 \mathrm{~V}$ & $\cdot / T$ & $1 / 0$ & $F+9$ & $\Lambda T / \Delta$ & يك \\
\hline $1 \cdot v \Delta$ & $1 \cdots$ & $1 \cdot 11$ & $1 / r$ & $\cdot f$ & $r q / 4$ & $r q / \lambda$ & 19. & Vif & $1 / \cdot$ & $1 \cdot 19$ & 9) & vif & FNA & ANG & دو \\
\hline fFr & $1 \cdots$ & $1 \Delta \lambda$ & $\cdot / V$ & $\cdot / V$ & 191 & 199 & gr & $M T$ & $\cdot / 9$ & NF & $1 / 9$ & $f * \%$ & 9999 & $\Delta M \Delta$ & سه وبيشتر \\
\hline & & & & & & & & & & & & & & & تحصيلات \\
\hline rqu & $1 \cdots$ & rMl & $T / 1$ & $\cdot / V$ & $r f / 0$ & TQT & $N r$ & gif & $\cdot r$ & $f / V$ & $\Delta f$ & rro & $\Delta 1 / 9$ & VGA & لبتدايى وكمتر \\
\hline$r \cdot r$ & $1 \ldots$ & $\mid f / 4$ & $\pi \cdot$ & $\cdot / 0$ & thig & $T Q 1$ & $1 \cdot / r$ & $1 / 9$ & $r \cdot \cdot$ & $11 / 1$ & $\Delta q$ & $r \cdot / V$ & $\Delta N G$ & $\Lambda \pi \mathrm{V}$ & راهنمايى \\
\hline Me & $1 \cdots$ & $1 \pi 9$ & fif & $\cdot \pi$ & $\pi / \cdot$ & $\pi / T$ & $1 . / 9$ & 99 & $1 / 9$ & $11 / V$ & $f / Y$ & $15 / 9$ & $\mathrm{fq} / \mathrm{V}$ & $\lambda T / \cdot$ & ديرستان \\
\hline$|1 r|$ & $1 \cdots$ & $\mid r / 4$ & 9. & $\cdot f f$ & raV & $|r| \mid$ & ING & gr & $\cdot / V$ & $V / 9$ & $f / f$ & 99 & $f F / Q$ & $1 \mathrm{~N} / \mathrm{G}$ & دييلم \\
\hline $1 \cdot v f$ & $1 \cdots$ & $11 /$ & GT & $\%$ & $\Gamma f / \Delta$ & $r f / \Delta$ & $r \cdot / \Lambda$ & $v \cdot$ & $\cdot 19$ & $\Delta / \Delta$ & $1 / r$ & $\pi$ & FNT & $\lambda T / V$ & بالاثر از دييلم \\
\hline & & & & & & & & & & & & & & & اشتغال \\
\hline rfre & $1 \ldots$ & MT & $\Delta \Delta$ & $\cdot f f$ & $r / v$ & $\pi / 1$ & $18 / 7$ & $v \cdot$ & $\cdot / 9$ & Vis & $f / 1$ & $1 \cdot 11$ & $f q / 1$ & AN/T & غيرشاغل \\
\hline$\Delta r \Lambda$ & $1 \cdots$ & $11 / 9$ & $f / r$ & $\%$ & $f \cdot / V$ & $f \cdot / V$ & $r \Delta \Lambda$ & $\Delta \varphi$ & $\cdot / f$ & $9 \pi$ & $\cdot / 9$ & $f / 1$ & $f+1$ & $\Lambda \Pi \wedge$ & شاغل \\
\hline & & & & & & & & & & & & & & & هزيندة خانوار \\
\hline$v \cdot 1$ & $1 \cdots$ & $\mid f / V$ & $V / r$ & $\cdot / 9$ & $\pi / T$ & $\pi / A$ & $\mid \mathrm{V} \cdot$ & Vir & 1/. & NT & $T / f$ & $9 / r$ & $f Q R$ & VN. & كمتر از . \\
\hline Var & $1 \cdots$ & $1 \pi 9$ & $\Delta 9$ & $\cdot / r$ & $r M / r$ & $r v / \Delta$ & IVIV & $N$ & "/1 & $N \cdot$ & $f / \cdot$ & $1 \cdot / r$ & $f q / 1$ & $1 \cdot 19$ & $\mid f q 9-1 \ldots$ \\
\hline$\Delta \Delta f$ & $1 \cdots$ & $11 / \mathrm{V}$ & Qf & $\cdot \pi$ & $|+f| \mid$ & $r f / T$ & $r y / 9$ & 99 & $\%$ & Vir & $M / T$ & $9 / V$ & FNG & $\Lambda T / 9$ & $1999-10 \ldots$ \\
\hline $1 \cdots 0$ & $1 \cdots$ & $1 \pi / 4$ & $r / r$ & $\cdot \pi$ & $T \Delta T$ & $r \Delta \Delta$ & $T F / Q$ & $\Delta T$ & w & 90 & $f / 1$ & Vig & $\mathrm{Fq} / \mathrm{.}$ & $\lambda f / \Delta$ & r... \\
\hline & & & & & & & & & & & & & & & منطقةُ سكونت \\
\hline 1.94 & $1 \cdots$ & $I r / \Lambda$ & $M$ & $\cdot / 1$ & TQT & TQR & TNG & $\Delta G$ & $\cdot / V$ & $\Delta / V$ & $r / f$ & $\Delta \varphi$ & FNG & $1 \pi / 9$ & شمالى \\
\hline $11 \cdot v$ & $1 \cdots$ & $11 / V$ & gif & $\cdot / \Delta$ & $\Gamma f / \Lambda$ & $r \Delta T$ & $1 Q 9$ & git & $\cdot / \mathrm{V}$ & $N \Delta$ & $\Delta \cdot$ & $1 . / 4$ & fag & $11 / 9$ & مركزى \\
\hline Aft & $1 \cdots$ & $1 \Delta T$ & gr & $\cdot / r$ & rq/ & rqf & 199 & NG & $1 / r$ & NI & 》 & $11 / 0$ & $f q / r$ & YNG & جنوى \\
\hline & & & & & & & & & & & & & & & قوميت \\
\hline ISAT & $1 \cdots$ & $11 / f$ & $F / V$ & $\cdot / r$ & rgif & rag & rFT & $\Delta Y$ & $\cdot / \Lambda$ & $\Delta \wedge$ & $r / 9$ & 999 & fVir & $1 M 9$ & فارس \\
\hline 91. & $1 \cdots$ & $1 \Delta \Delta$ & $Q \Lambda$ & $\cdot / f$ & TNA & rqu & lQfF & $V / \Delta$ & $\cdot / 9$ & $9 / \Delta$ & T/T & 11). & $f q / Q$ & VNY & أذرى \\
\hline$r \cdot r$ & $1 \cdots$ & INT & gif & $\bullet$ & $M V$ & $\mathrm{HTV}$ & LQF & $N F$ & $\cdot \cdot$ & $\Delta / 9$ & $\Pi \cdot$ & $N 9$ & $f / / 9$ & VQT & كيلك ومازتيدرائى \\
\hline $1 \cdot 0$ & $1 \cdots$ & $\mid f / K$ & $\Delta Y$ & $\%$ & TNG & TNG & $|N|$ & $V / \Delta$ & $\%$ & NG & $1 / 9$ & IQT & $\Delta l / f$ & $1 * \%$ & 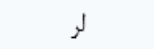 \\
\hline $11 \pi$ & $1 \cdots$ & $N \cdot$ & gr & $\cdot / 9$ & TNT & rqu & $r \cdot f$ & $11 / f$ & $r / V$ & 109 & $T / V$ & $\Gamma / \Delta$ & $\Delta 99$ & $N Q \Lambda$ & كرد/ساير \\
\hline & & & & & & & & & & & & & & & وضعيت مهاجرت \\
\hline$r \cdot A r$ & $1 \cdots$ & $11 / 9$ & $Q \Lambda$ & $\cdot / r$ & $r f / f$ & $r f / V$ & THT & gr & $\cdot / \Lambda$ & 99 & $r T$ & Vif & fyig & $\Lambda T / r$ & غيرمهاجر \\
\hline $9 \Delta \Delta$ & $1 \cdots$ & 19. & $f / 1$ & $\cdot r$ & $r y / r$ & ri/s & $|9|$ & Vil & $\cdot / \Lambda$ & $9 / r$ & +1 & $1 \mathrm{l} \cdot$ & FNT & vqu & مهلجر ازٔشهر \\
\hline rVD & $1 \cdots$ & $\mid f / 0$ & $M G$ & $\cdot / f$ & $r \cdot / \Delta$ & $r \cdot / 9$ & $11 / r$ & $q / \Delta$ & $\cdot / \Lambda$ & $9 / 1$ & $M 9$ & 198 & $\Delta \cdot / 9$ & $\Lambda 1 / \Lambda$ & مهاججر أر روستا \\
\hline$r+1 r$ & $1 .$. & $\mid r / *$ & $\Delta / r$ & $* / r$ & $\mathrm{rr} / \mathrm{f}$ & rr/v & $T \cdot / 9$ & $g / V$ &.$/ 1$ & $V / f$ & $r / \Delta$ & १/. & $\mathrm{FN} \cdot$ & $\Lambda \mathrm{I} / \mathrm{V}$ & كل \\
\hline
\end{tabular}


فرزند در نوسان است. همينطور بيشترين استفاده از كاندوم در

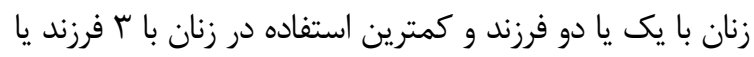

$$
\text { بيشتر (4 درصد) ديده مىشود. }
$$

شكل r شايعترين روشهاى استفادهده را بر حسب تعداد

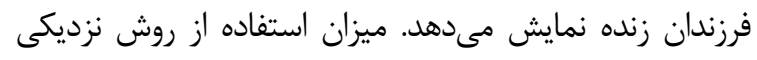

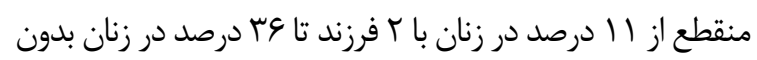

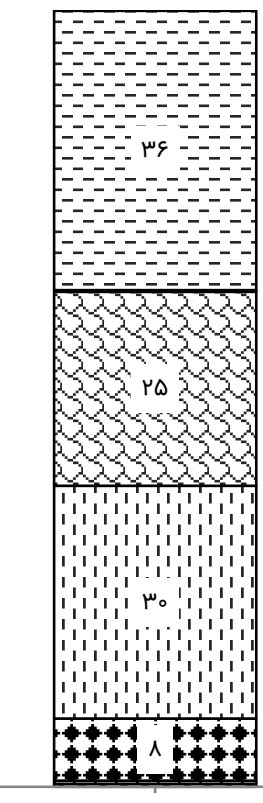

نمودار ץ. الكَوى استفاده از روشهاى بيشعَيرى از باردارى (درصد) برحسب تعداد فرزندان زنده در ميان زنان متاهل ها-9q ساله،

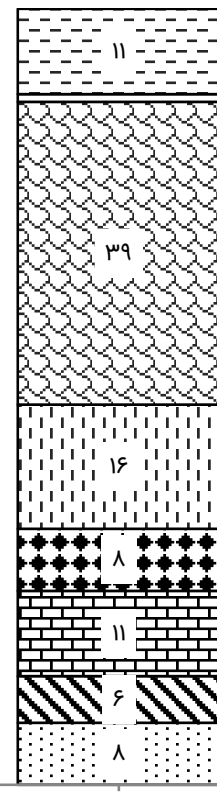

r

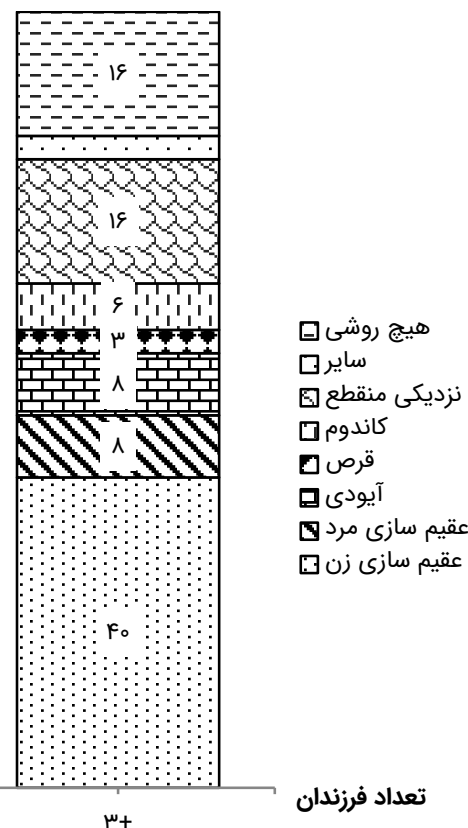

\section{عقيم سازى مردى}

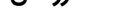
تهران سو (درصن برحس

درصد در سال سوس| كاهش يافته است. همجنين استفاده از

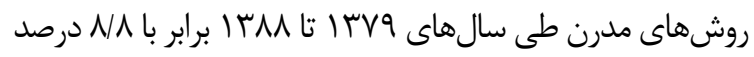

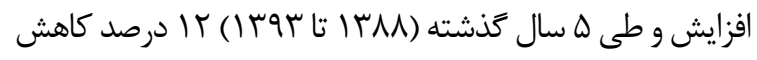

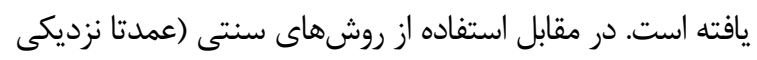

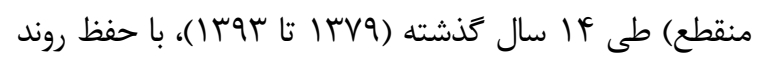

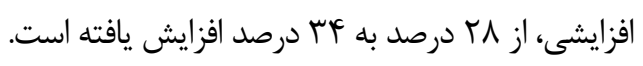

روند استفاده از روشهاى يِيشگيرى از باردارى

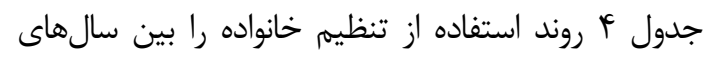
(TV9

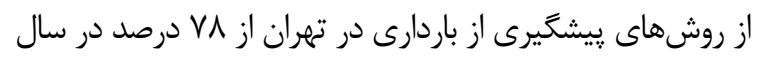

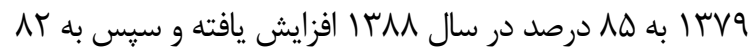

جدول F. توزيع درصد زنان متأهل بر حسب استفاده از روشهاى كنونى ييشغيرى از باردارى در سالهاى

\begin{tabular}{|c|c|c|c|}
\hline سال זarו & 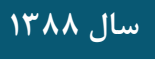 & سال & روش بيشَيرى از باردارى \\
\hline$\wedge 1 / \vee$ & $\Lambda \Delta / \Gamma$ & $V V / q$ & هملهُ روشها \\
\hline$F \wedge /$. & $\Delta F / F$ & $\Delta \cdot 1 \cdot$ & روشهاى مدرن \\
\hline $9 / \cdot$ & $1 \cdot 10$ & $11 / \pi$ & 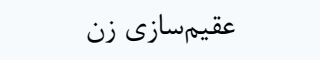 \\
\hline$r / \Delta$ & $q / 4$ & $\Delta / \cdot$ & عقيمسازى مرد \\
\hline$V / F$ & $\mid r / \Delta$ & $\mid \Delta / \cdot$ & آيودى \\
\hline$\cdot / r$ & $\cdot 19$ & $\cdot 11$ & نوريلنت \\
\hline$\cdot 19$ &. & $\cdot / V$ & تززيق \\
\hline $9 / V$ & N/9 & $11 / 0$ & 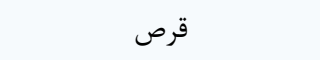 \\
\hline$r \cdot 19$ & $10 / \Delta$ & $9 / 4$ & 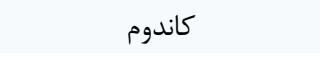 \\
\hline
\end{tabular}




\begin{tabular}{|c|c|c|c|}
\hline سال זهץ" & سال Ir^1 & سال Irva & روش بيشعَيرى از باردارى \\
\hline 然/V & $r \cdot / 9$ & TV/G & روشهاى سنتى \\
\hline$r \mu / \epsilon$ & $r \cdot / r$ & $r \varepsilon / 9$ & نزديكى منقطع \\
\hline$\cdot \pi$ & $\cdot / \mathrm{V}$ & $\cdot / \mathrm{V}$ & دورة مطمئن \\
\hline $11 / \pi$ & $1 F / V$ & $r r / F$ & هيج روشى \\
\hline $1 \cdots 1$ & $1 \cdots /$ & $1 \cdot . /$ & كل \\
\hline
\end{tabular}

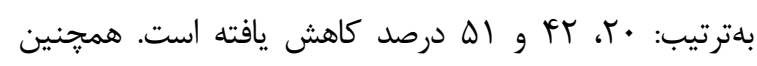

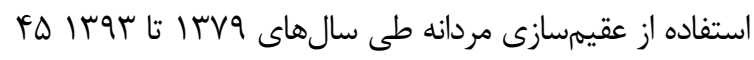
درصد كاهش يافته است.
يافتهها بهتفكيك روشهاى ييشخيرى در جدول أ و نمودار

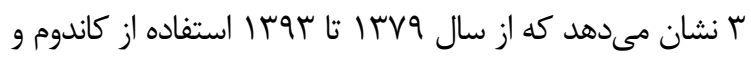

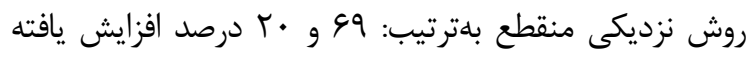

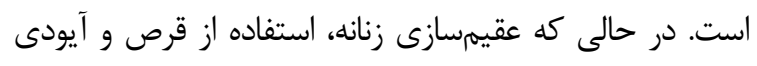

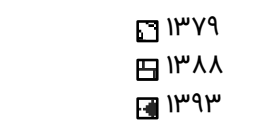

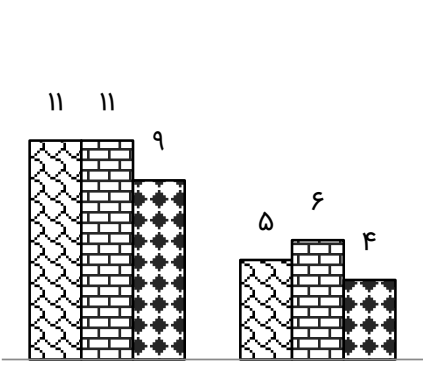

عقيم سازى مرد عقيم سازى زن

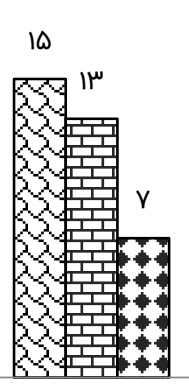

آيودى

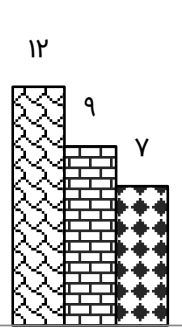

قرص - م

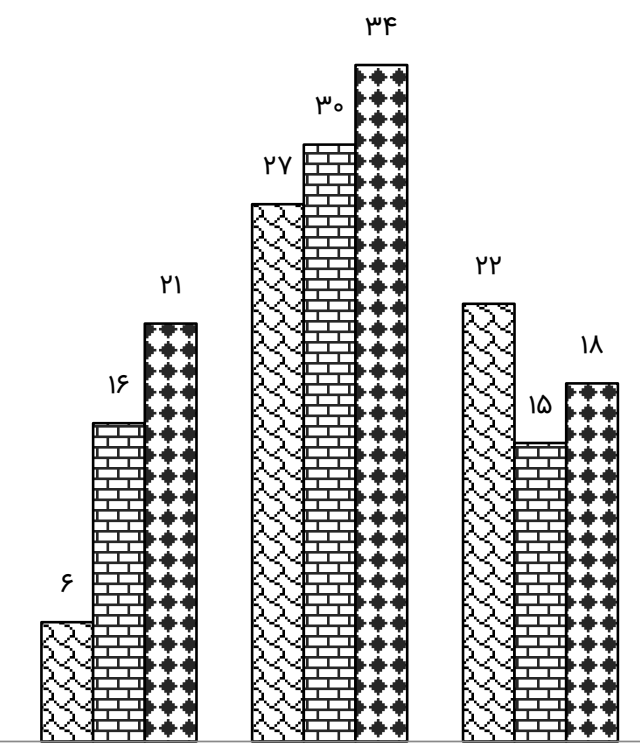

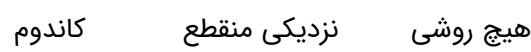

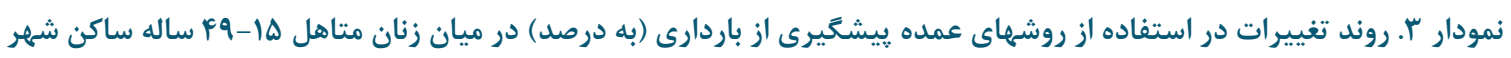

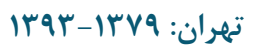

يافتهاى يزوهش نشان مىدهد كه از سال IrV9 تا

بو ا استفاده از كاندوم و روش نزديكى منقطع بلهترتيب: 99

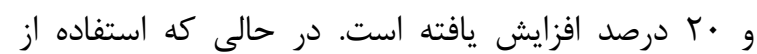

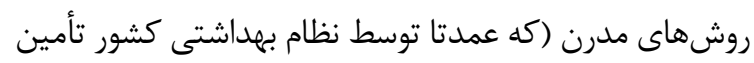

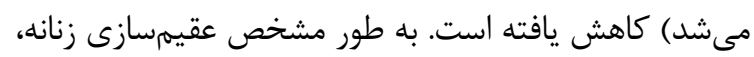

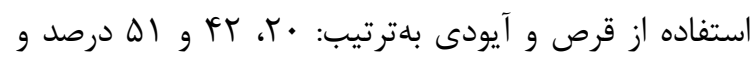

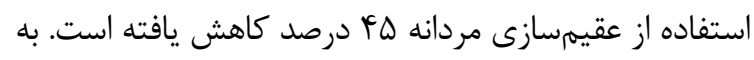

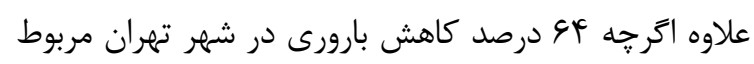

بحث

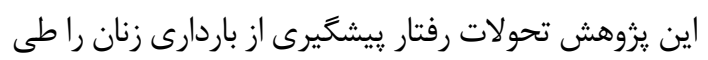
سالهاى

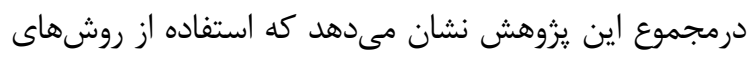

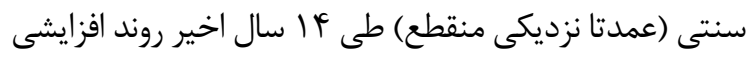

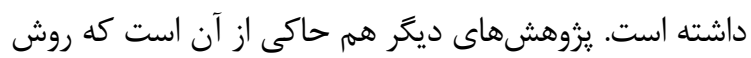

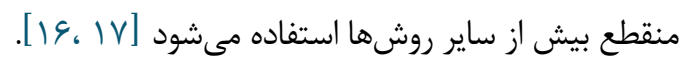




$$
\text { צو روند و الكوى ريشخيرى از باردارى در تهران }
$$

4. Conde-Agudelo A, Rosas-Bermúdez A, Kafury-Goeta AC. Birth Spacing and Risk of Adverse Perinatal Outcomes: A Meta-Analysis. Jama. 2006 Apr 19;295(15):1809-23.

[DOI:10.1001/jama.295.15.1809] [PMID]

5. Haq A, Lallar M, Akhter S, Baba YZ, Ahmad J, Hamid MA. Interpregnancy Interval Raise Odds of Adverse Perinatal Outcome in High Fertility Region Mewat, Haryana. International Journal of Reproduction, Contraception, Obstetrics and Gynecology. 2014;3(3):598-603. 1770.ijrcog20140934]

6. Hall KS, Steinberg JR, Cwiak CA, Allen RH, Marcus SM. Contraception and Mental Health: a Commentary on the evidence and principles for practice. American journal of obstetrics and gynecology. 2015 Jun 1;212(6):740-6. [DOI:10.1016/j.ajog.2014.12.010] [PMID] [PMCID]

7. Ahmed S, Li Q, Liu L, Tsui AO. Maternal Deaths Averted by Contraceptive Use: an Analysis of 172 Countries. The Lancet. 2012 Jul 14;380(9837):111-25. [DOI:10.1016/S0140-6736(12)60478-4]

8. Erfani A, McQuillan K. Rapid Fertility Decline in Iran: Analysis of Intermediate Variables. Journal of Biosocial Science. 2008 May;40(3):459-78. [DOI:10.1017/S002193200700243X] [PMID]

9. Erfani A. Curbing Publicly-Funded Family Planning Services in Iran: who Is Affected?. J Fam Plann Reprod Health Care. 2017 Jan 1;43(1):37-43. [DOI:10.1136/jfprhc-2015-101383] [PMID]

10. Erfani A. Curbing Family Planning in Iran: an Appraisal of Bill 446. BMJ Sexual \& Reproductive Health. 2015 Oct 1;41(4):317. [DOI:10.1136/ifprhc2015-101254] [PMID]

11. Erfani A. Tehran Survey of Fertility, 2014: Final Report. Tehran, Iran: National Population Studies and Comprehensive Management Institute, Ministry of Science. Research, and Technology. 2015.

12. Erfani A. Tehran Survey of Fertility, 2009: Final Report, Tehran, Iran: Population Studies and Research Center in Asia and Pacific, 2010. (Persian)

13. Ministry of Health and Medical Education (MOHME). Iran Demographic nd Health Survey 2000 (IDHS). Tehran: MOHME, Iran. 2000.

14. Erfani A. Induced Abortion in Tehran, Iran: estimated rates and correlates. International Perspectives on Sexual \& Reproductive Health. 2011 Sep 1;37(3): 134142 [DOI:10.1363/3713411] [PMID]

15. Erfani A, McQuillan K. The Changing Timing of Births in Iran: an Explanation of the Rise and Fall in Fertility after the 1979 Islamic Revolution. Biodemography and social biology. 2014 Jan 2;60(1):67-86. [DOI:10.1080/19485565.2014.899428] [PMID]

16. Bakht R, Omidi A. Study of Contraceptive Methods Use before the First Pregnancy. Scientific Journal of Hamadan Nursing \& Midwifery Faculty. 2008 Nov $15 ; 16(2): 32-42$.
17. Ahmad Shirvani M, Omidian A. Utilization Rate of Natural Family Planning and its Related Factors. Journal of hayat. 2007 Jun 15;13(3):37-47.

18. Rastak L. Correlation between socio-demographic characteristics and contraceptive methods. J Shahrekord Univ Med Sci. 2005; 7 (2) :21-28.(Persian)

19. Amiri P, Amouzegar A, Gharibzadeh S, Kazemian E, Ramezani Tehrani F, Azizi F. Socio-Behavioral and Reproductive Determinants Associated with Contraceptive Method Choice Among Tehranian Women: Tehran Lipid and Glucose Study. Research in Medicine. 2014 Aug 1;38(2):111-9.

20. Erfani A. Levels, Trends and Correlates of Abortion in Tehran, Iran: 2009-2014. International perspectives on sexual and reproductive health. 2016 Jun 1;42(2):93101. [DOI:10.1363/42e1316] [PMID]

21. Erfani A, Shojaei J. New Evidence on Induced Abortion in Tehran, Iran: Rates, Causes, and Changes. The Iranian Journal of Obstetrics, Gynecology and Infertility. 2018;21(3):64-77.

22. Erfani A. Levels, Trends, and Determinants of Unintended Pregnancy in Iran: the Role of Contraceptive Failures. Studies in Family Planning. 2013 Sep;44(3):299-317. [DOI:10.1111/j.17284465.2013.00359.x] [PMID]

23. Zaheri F, Ranaie F, Karimeh R, Shahoi R. Unwanted Pregnancy and Associated Factors Among Pregnant Women Who Referred to Sanandaj Health Centers in 2011. The Iranian Journal of Obstetrics, Gynecology and Infertility. 2015;17(132):10-5.

24. Erfani A, Yuksel-Kaptanoglu I. The Use of Withdrawal Among Birth Limiters in Iran and Turkey. Studies in Family Planning. 2012 Mar;43(1):21-32. [DOI:10.1111/j.1728-4465.2012.00299.x] [PMID]

25. Nazarpour S, Âzimi H. Study on the Rate of Using Different Family Planning Methods and Attitudes of Under 25 Years Married Females Towards Family Planning Referring to West Province Health Center of Mazandaran in 2001. Journal of Mazandaran University of Medical Sciences. 2002 Dec 15;12(37):46-55.

26. Pouransary Z, Sheikh Z, Eshrati B, Kamali P. Prevalence of Contraceptive Use and the Importance of the Husband's Cooperation in Married Women in Iranshahr, 2005. Iranian Journal of Epidemiology. 2008 Feb 15;3(3):55-60. 